\begin{tabular}{|c|c|}
\hline Title & Three valued magnetic tunnel junction for nonvolatile ternary content addressable memory application \\
\hline Author(s) & Uemura, Tetsuya; Y amamoto, Masafumi \\
\hline Citation & $\begin{array}{l}\text { Journal of A pplied Physics, 104(12), } 123911 \\
\text { https://doi.org/10.1063/1.3054174 }\end{array}$ \\
\hline Issue Date & $2008-12-23$ \\
\hline Doc URL & http:/hdl.handle.net/2115/50623 \\
\hline Rights & $\begin{array}{l}\text { Copyright } 2008 \text { A merican Institute of Phy sics. This article may be downloaded for personal use only. A ny other use } \\
\text { requires prior permission of the author and the A merican Institute of Physics. The following article appeared in J. A ppl. } \\
\text { Phys. 104, 123911 (2008) and may be found at https://dx.doi.org/10.1063/1.3054174 }\end{array}$ \\
\hline Type & article \\
\hline File Information & JAP104_123911.pdf \\
\hline
\end{tabular}

Instructions for use 


\title{
Three-valued magnetic tunnel junction for nonvolatile ternary content addressable memory application
}

\author{
Tetsuya Uemura ${ }^{a}$ and Masafumi Yamamoto \\ Division of Electronics for Informatics, Hokkaido University, Kita 14, Nishi 9-Sapporo, Hokkaido 060-0814, \\ Japan
}

(Received 6 May 2008; accepted 11 November 2008; published online 23 December 2008)

\begin{abstract}
Three-valued epitaxial $\mathrm{Co}_{50} \mathrm{Fe}_{50} / \mathrm{MgO} / \mathrm{Co}_{50} \mathrm{Fe}_{50}$ magnetic tunnel junctions (MTJs) were developed for nonvolatile ternary content addressable memory (TCAM) application. Four remanent magnetization states in the single-crystalline $\mathrm{Co}_{50} \mathrm{Fe}_{50}$ electrode, due to cubic anisotropy with easy axes of the $\langle 110\rangle$ directions, result in four possible angular-dependent tunnel magnetoresistance ratios. Three states selected from among the four states were separated by more than $56 \%$ at room temperature. Analysis of the asteroid curve for $\mathrm{Co}_{50} \mathrm{Fe}_{50}$ indicated that a magnetic field along $22.5^{\circ}$ from the $\langle 110\rangle$ directions made it possible to change the magnetization direction of the selected cell without disturbing those of the half-selected cells in the MTJ arrays. A nonvolatile TCAM cell using the three-valued MTJ for three-level data storage was proposed, and its operation was verified by circuit simulation. The TCAM cell reduces the device count to 5, a value $1 / 3$ that of conventional complementary metal-oxide semiconductor-based TCAMs. (c) 2008 American Institute of Physics. [DOI: $10.1063 / 1.3054174]$
\end{abstract}

\section{INTRODUCTION}

Magnetic tunnel junctions (MTJs) with multilevel signal storing capability are attractive for high-density memories, such as magnetic random access memories (MRAMs) and/or content addressable memories (CAMs). As ferromagnets become smaller, the switching field-i.e., the write signal current-increases, so large dissipation power and/or a large peripheral circuit are required. Multilevel signal storing is a promising approach to increase the storage density without reducing the size of the ferromagnet. Although several multistate MTJs or giant magnetoresistance (GMR) devices have been reported, ${ }^{1-4}$ these devices are not adequate for practical use in terms of their MR ratio or operating temperature. Zheng et $\mathrm{al}^{3}{ }^{3}$ reported a four-state GMR device that uses a $\mathrm{NiFe} / \mathrm{CoFe} / \mathrm{Cu}$ system with a MR ratio of approximately $3.0 \%$. Tanaka et $a .^{4}$ reported a four-state MTJ that uses a $\mathrm{NiMnSb}$ system with a tunnel MR (TMR) ratio of approximately $4.5 \%$ at $77 \mathrm{~K}$.

Very high TMR ratios of more than $100 \%$ were recently achieved at room temperature (RT) in MTJs with epitaxial or highly oriented ferromagnets and an $\mathrm{MgO}$ tunnel barrier. ${ }^{5-11}$ This enables a practical multistate MRAM. We fabricated a multistate MTJ using a fully epitaxial $\mathrm{Co}_{50} \mathrm{Fe}_{50} /$ $\mathrm{MgO} / \mathrm{Co}_{50} \mathrm{Fe}_{50}$ structure and observed four-state TMR values. ${ }^{12}$ Although the TMR ratio of the fabricated MTJ was as large as $145 \%$ at RT as a binary mode, it was reduced to $20 \%$ as a four-state mode. In our current study, we developed a three-state MTJ with TMR ratios of more than 56\% using the multistate $\mathrm{Co}_{50} \mathrm{Fe}_{50} / \mathrm{MgO} / \mathrm{Co}_{50} \mathrm{Fe}_{50}$ MTJ by selecting three states of the four states. Furthermore, we designed and proposed a ternary CAM (TCAM). The proposed TCAM cell uses the three-state MTJ for three-level data storage and an

${ }^{a)}$ Electronic mail: uemura@ist.hokudai.ac.jp. inverter with a negative differential resistance (NDR) load for ternary data matching. In Sec. II, we describe the fabrication and characterization of the multistate MTJs. Based on the analysis of the asteroid curve for a cubic anisotropy, we describe a novel writing method using a combination of orthogonal magnetic fields, which enables us to change the magnetization direction of the selected cell without disturbing those of the half-selected cells. In Sec. III, we describe the cell architecture of the TCAM and verify its operation through circuit simulations based on the device characteristics of the fabricated multistate MTJs.

\section{MULTISTATE MTJ}

\section{A. Angular-dependent TMR}

The TMR of the MTJ depends on the angle $\theta$ between the magnetization vector of the free layer and that of the pinned layer. Based on Julliere's model, the TMR value $R$ can be written as

$$
R=R_{\mathrm{P}}\left(1+\frac{P_{1} P_{2}}{1+P_{1} P_{2} \cos \theta}(1-\cos \theta)\right),
$$

where $P_{1}$ and $P_{2}$ are the respective spin polarizations of the free layer and the pinned layer, and $R_{\mathrm{P}}$ is the TMR value when both magnetization vectors are parallel. Since an epitaxial $\mathrm{Co}_{50} \mathrm{Fe}_{50}$ electrode has four in-plane remanent magne-

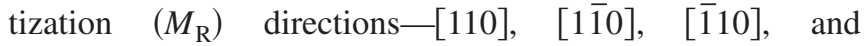
[1]10] — due to the cubic magnetocrystalline anisotropy, $\theta$ takes four possible values- $\phi_{0}, \phi_{0}+90^{\circ}, \phi_{0}+180^{\circ}$, and $\phi_{0}$ $+270^{\circ}$-in the absence of an applied magnetic field, where $\phi_{0}$ is the angle of the magnetization vector of the pinned layer with respect to the $\mathrm{Co}_{50} \mathrm{Fe}_{50}[110]$ direction. Figure 1 plots the calculated TMR values as a function of $\theta$. We used $P_{1}=P_{2}=0.648$ for the spin polarizations, as determined from the TMR value obtained experimentally in the fully epitaxial 


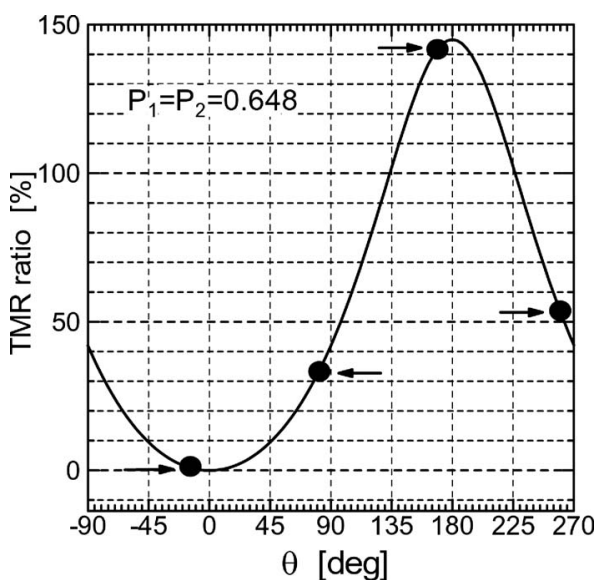

FIG. 1. Calculated TMR ratio as a function of $\theta$. Solid circles indicate four possible $\theta$ values $\phi_{0}, \phi_{0}+90^{\circ}, \phi_{0}+180^{\circ}$, and $\phi_{0}+270^{\circ}$ for $\phi_{0}$ set to a slightly smaller value than $0^{\circ}$, where $\phi_{0}$ is the angle of the magnetization vector of the pinned layer.

$\mathrm{Co}_{50} \mathrm{Fe}_{50} / \mathrm{MgO} / \mathrm{Co}_{50} \mathrm{Fe}_{50}$ MTJ used in this study. The solid circles in Fig. 1 indicate four possible $\theta$ values $-\phi_{0}, \phi_{0}$ $+90^{\circ}, \phi_{0}+180^{\circ}$, and $\phi_{0}+270^{\circ}$ - for a $\phi_{0}$ set to be slightly smaller than $0^{\circ}$. These four possible values provide four-state TMR values. If $\phi_{0}$ is set to $0^{\circ}$, three different TMR values are obtained, since two of the four states among $\phi_{0}+\alpha(\alpha$ $=0^{\circ}, 90^{\circ}, 180^{\circ}$, and $270^{\circ}$ ) provide an equal TMR value.

\section{B. Device fabrication}

Figure 2 depicts the layer structure of the fabricated MTJ. Layers of (from the top) $\mathrm{Ru}(5 \mathrm{~nm}) / \mathrm{IrMn}(10) /$ $\mathrm{Co}_{90} \mathrm{Fe}_{10}(2) / \mathrm{Ru}(0.8) / \mathrm{Co}_{50} \mathrm{Fe}_{50}(3) / \mathrm{MgO}(1.7) / \mathrm{Co}_{50} \mathrm{Fe}_{50}(50) /$ $\mathrm{MgO}(10)$ were grown on a $\mathrm{MgO}(001)$ substrate. Each layer was successively deposited in an ultrahigh vacuum chamber (with a base pressure of about $6 \times 10^{-8} \mathrm{~Pa}$ ) through the combined use of magnetron sputtering and electron beam evaporation. All layers except for the $\mathrm{MgO}$ layers were deposited at RT using magnetron sputtering. The $\mathrm{MgO}$ buffer layer and $\mathrm{MgO}$ tunnel barrier were deposited by electron beam evaporation at $400{ }^{\circ} \mathrm{C}$ and $\mathrm{RT}$, respectively.

The $10 \times 10 \mu \mathrm{m}^{2}$ MTJs were fabricated using photolithography and Ar ion milling. After fabrication, the MTJs were annealed at $300{ }^{\circ} \mathrm{C}$ for 60 min under a magnetic field of $5 \mathrm{kOe}$ in a vacuum of $5 \times 10^{-2} \mathrm{~Pa}$. To obtain four TMR

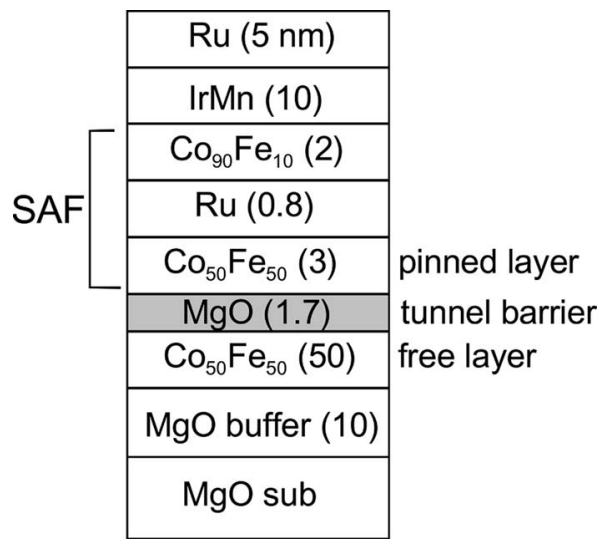

FIG. 2. Layer structure of the fabricated MTJ.
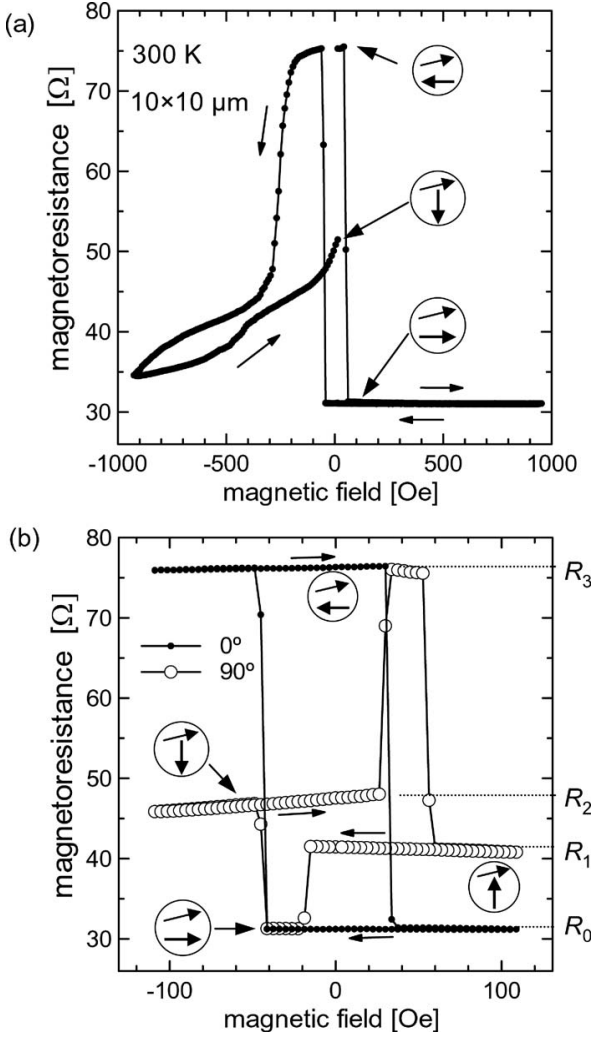

FIG. 3. (a) Major loop and (b) minor loops of TMR characteristics for a fabricated MTJ. Magnetization configurations for each state were depicted.

values, the magnetization direction of the upper $\mathrm{Co}_{50} \mathrm{Fe}_{50}$ layer was pinned through exchange biasing along a direction approximately $10^{\circ}$ off that of the $\mathrm{Co}_{50} \mathrm{Fe}_{50}$ [110]. A synthetic antiferromagnetic $(\mathrm{SAF})$ trilayer structure consisting of $\mathrm{Co}_{90} \mathrm{Fe}_{10}(2) / \mathrm{Ru}(0.8) / \mathrm{Co}_{50} \mathrm{Fe}_{50}(3)$ was used to lower the net saturation magnetization in the pinned structure with IrMn, enabling a high exchange bias field $H_{\text {ex }}$. The TMR characteristics of the fabricated devices were measured through a dc four-probe method at RT.

\section{MR characteristics}

Figure 3(a) shows a major loop of the TMR curve for a fabricated device with a size of $10 \times 10 \mu \mathrm{m}^{2}$. The magnetic field $H$ was applied from -950 to 950 Oe along the pinned direction. The maximum TMR ratio, defined by $\left(R_{\mathrm{AP}}\right.$ $\left.-R_{\mathrm{P}}\right) / R_{\mathrm{P}}$, was approximately $145 \%$ at $\mathrm{RT}$, where $R_{\mathrm{AP}}$ and $R_{\mathrm{P}}$ were the TMR values with $\theta$ of $180^{\circ}$ and $0^{\circ}$, respectively. A relatively large $H_{\mathrm{ex}}$ of approximately 150 Oe was obtained due to the SAF structure.

Figure 3(b) shows minor loops of the TMR curves for the same device with two different directions. The $H$ ranging from -120 to 120 Oe was applied along [110] and [110] directions. Note that the magnetization vector of the $\mathrm{Co}_{50} \mathrm{Fe}_{50}$ pinned layer was always fixed in the same direction at $|H|=0\left(10^{\circ}\right.$ off [110]) due to a large $H_{\text {ex }}$. The TMR at $|H|=0$ showed four distinct values-31, 41, 49, and $76 \Omega$-each separated by more than $20 \%$. These values cor- 


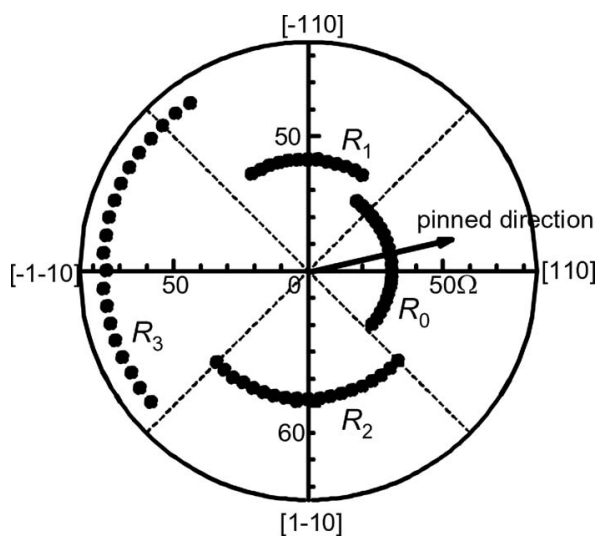

FIG. 4. Polar plot of MR in the absence of a magnetic field.

respond to the states where the $M_{\mathrm{R}}$ lies for the [110], [110], [1 $1 \overline{1} 0]$, and [ $\overline{1} \overline{1} 0]$ directions, respectively. We refer to these four TMR values as $R_{0}, R_{1}, R_{2}$, and $R_{3}$.

Figure 4 shows a polar plot of the TMR at $|H|=0$. The angle of the polar plot corresponds to the direction of $H$, which was applied before measurement with various directions from $0^{\circ}$ to $360^{\circ}$ in steps of $5^{\circ}$ in the (001) plane to set the direction of $M_{\mathrm{R}}$. Because the pinned direction lay $10^{\circ}$ off the [110] direction, the $R_{1}$ was $20 \%$ smaller than the $R_{2}$. If $R_{0}, R_{2}$, and $R_{3}$ are used as a three-state MTJ, the TMR values are separated by more than $56 \%$.

\section{Selective write operation in MTJ array}

In the MTJ arrays, each level must be written without disturbing other unselected cells. A combination of the orthogonal fields $H_{W x}$ and $H_{W y}$ is used for the selective writing in the conventional MRAM. In this subsection, selective writing for the multistate MTJ array is considered. We defined the $x$ and $y$ directions as $-22.5^{\circ}$ and $67.5^{\circ}$ from the [110] direction, as shown in Fig. 5(a). Figure 5(b) shows the calculated asteroid curve of the free layer with cubic magnetocrystalline anisotropy. Let us consider the case when $H_{W x}$ and $H_{W y}$ with a strength between $K_{1} / 2 \sqrt{2}$ and $K_{1} / 2$ are applied, where $K_{1}$ is a cubic anisotropy constant divided by the saturation magnetization. The combination of $H_{W x}$ and $H_{W y}$ is then applied to the selected cell, while either $H_{W x}$ or $H_{W y}$ is applied to the half-selected cell. Application of either $H_{W x}$ or $H_{W y}$ does not switch any magnetization direction, while the $H_{W x}+H_{W y}$ field, whose strength is between $K_{1} / 2$ and $\sqrt{2} K_{1} / 2$, can switch the magnetization vector with the [1]0]

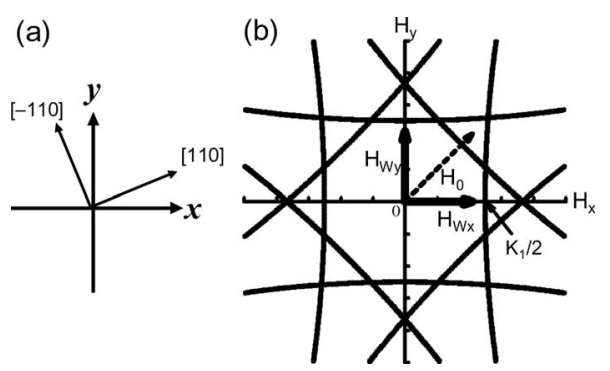

FIG. 5. (a) Definition of $x$ and $y$ directions and (b) calculated asteroid curve for $\mathrm{Co}_{50} \mathrm{Fe}_{50}$ with cubic magnetocrystalline anisotropy.
TABLE I. Write data versus sequence of write field.

\begin{tabular}{lc}
\hline \hline Write data & Sequence of write field \\
\hline$R_{0}$ & $\boldsymbol{H}_{1} \rightarrow \boldsymbol{H}_{3} \rightarrow \boldsymbol{H}_{2} \rightarrow \boldsymbol{H}_{0}$ \\
$R_{1}$ & $\boldsymbol{H}_{3} \rightarrow \boldsymbol{H}_{2} \rightarrow \boldsymbol{H}_{0} \rightarrow \boldsymbol{H}_{1}$ \\
$R_{2}$ & $\boldsymbol{H}_{0} \rightarrow \boldsymbol{H}_{1} \rightarrow \boldsymbol{H}_{3} \rightarrow \boldsymbol{H}_{2}$ \\
$R_{3}$ & $\boldsymbol{H}_{2} \rightarrow \boldsymbol{H}_{0} \rightarrow \boldsymbol{H}_{1} \rightarrow \boldsymbol{H}_{3}$ \\
\hline \hline
\end{tabular}

direction (the $R_{2}$ state) to the [110] direction (the $R_{0}$ state). Likewise, the $-H_{W x}+H_{W y}$ field can switch only an $R_{0}$ state to an $R_{1}$ state, and so on. Based on this scheme, we propose a novel writing method as follows. Let us define the write fields $H_{W x}+H_{W y},-H_{W x}+H_{W y}, H_{W x}-H_{W y}$, and $-H_{W x}-H_{W y}$ as $H_{0}, H_{1}, H_{2}$, and $H_{3}$. To write the $R_{0}$ state from any other states without disturbing other half-selected cells, the write fields of $H_{1}, H_{3}, H_{2}$, and $H_{0}$ are applied in order. Table I summarizes the sequence of the applied field in a write operation.

\section{TERNARY CONTENT ADDRESSABLE MEMORY}

In this section, we describe a novel nonvolatile TCAM using the multistate MTJ. The CAMs compare search data against a table of stored data and return the address of the matching data. Many multilevel CAMs have been proposed using floating-gate metal-oxide-semiconductor field-effect transistor (MOSFETs), ${ }^{13}$ ferroelectric capacitors, ${ }^{14}$ metal ferroelectric semiconductor FETs, ${ }^{15}$ or single-electron transistors (SETs) (Ref. 16) in order to achieve higher density and/or higher search speed than conventional binary CAMs. As a special case of multilevel CAMs, Degawa et al. ${ }^{17}$ recently proposed a TCAM using SETs and showed that the SET-based cell architecture reduced the number of transistors to $1 / 3$ compared with the conventional complementary MOS (CMOS)-based one. In addition to " 0 " and "1," the TCAM also stores a "do not care" bit ("X"). With this don't care bit stored in the TCAM cell, the matching result is always "match" regardless of the search data. Thus, TCAMs are

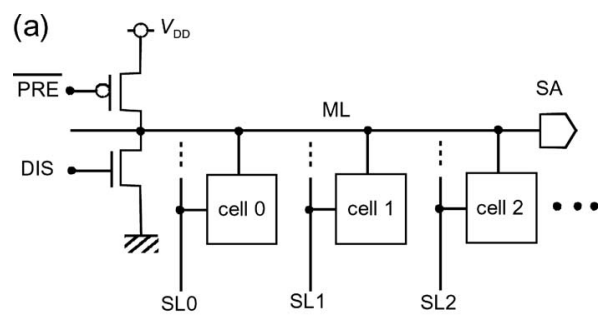

(b)

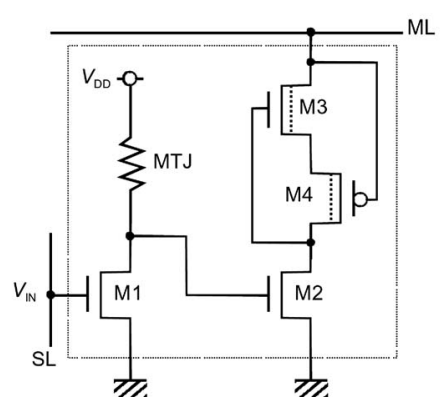

FIG. 6. (a) Architecture and (b) cell structure of proposed TCAM. 
(a)

(b)

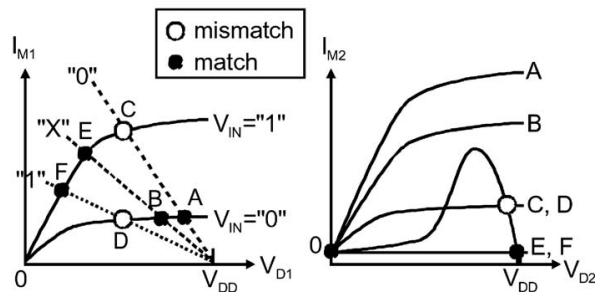

FIG. 7. Schematic load-line diagram of (a) first inverter and (b) second inverter.

widely used in network routers, which require high-speed address translation with IP address masking. In our current study, we propose a novel TCAM cell using the multistate MTJ described in Sec. II.

\section{A. MTJ-based TCAM architecture}

Figures 6(a) and 6(b) illustrate the architecture and cell structure of the proposed TCAM. The TCAM consists of memory cells, search lines, match lines (MLs), and sense amplifiers. The memory cell consists of a two-stage inverter with a MTJ load and a NDR load. The key features of the proposed cell are (i) it uses the MTJ for multistate data storage, enabling low-power operation due to the nonvolatility and (ii) it uses a NDR element as a load in the second inverter, making it possible to reduce the number of devices due to the increased functionality. Since the memory cell uses one MTJ and four MOSFETs, the number of devices can be reduced to $1 / 3$ that of a conventional CMOS-based TCAM cell consisting of a two-bit static RAM (SRAM) cell and four matching transistors. ${ }^{17}$ Moreover, since the MTJbased cell is nonvolatile, lower stand-by power consumption is possible.

Figure 7(a) shows a schematic load-line diagram of the first inverter of the TCAM cell. Since the search data are input to the gate terminal of M1, the transistor characteristics are changed depending on the search data, 0 or 1 . The resistance value of the MTJ was, on the other hand, changed by the stored data: 0,1 , or X. The black circles labeled "A," "B," "E," and "F" in Fig. 7(a) indicate match conditions, including the don't care condition, while the white circles labeled " $C$ " and " $D$ " indicate "mismatch" conditions. The output of the first inverter is connected to the second inverter.

Figure 7(b) shows a schematic load-line diagram of the second inverter. Note that the load element consisting of a depletion-type n-channel MOS (NMOS) (M3) and a depletion-type p-channel MOS (PMOS) (M4) exhibits NDR characteristics. ${ }^{18}$ The transistor characteristics of the M2 depend on the output of the first inverter. The labels A, B, C, D,

TABLE II. SPICE parameters for MOSFETs.

\begin{tabular}{lccrl}
\hline \hline Write data & M1 & M2 & M3 & M4 \\
\hline$t_{\text {ox }}(\mathrm{nm})$ & 4.0 & 4.0 & 4.0 & 4.0 \\
$V_{\text {th }}(\mathrm{V})$ & 0.4 & 0.4 & -0.4 & 0.42 \\
$L(\mu \mathrm{m})$ & 0.2 & 0.2 & 0.2 & 0.2 \\
$W(\mu \mathrm{m})$ & 1.0 & 1.0 & 2.0 & 2.0 \\
\hline \hline
\end{tabular}

(a)

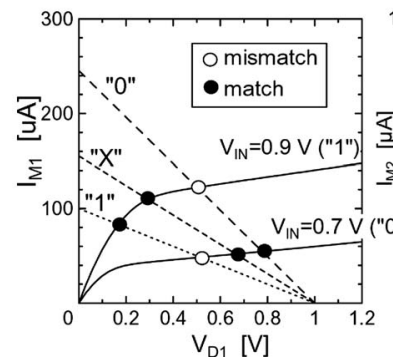

(b)

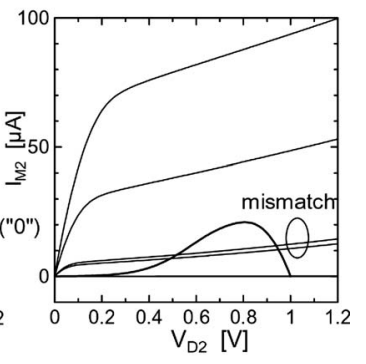

FIG. 8. SPICE simulation results for (a) first inverter and (b) second inverter of TCAM cell.

$\mathrm{E}$, and $\mathrm{F}$ shown in Fig. 7(b) correspond to the operating points labeled by the same symbols in Fig. 7(a). The peak current of the NDR load is set between the drain current labeled $\mathrm{B}$ and that labeled $\mathrm{C}$ or D, as shown in Fig. 7(b). If a mismatch condition is satisfied, a large current flows ( $\mathrm{C}$ or D), while almost no current flows if a match condition is satisfied (A, B, E, or F). Thus, the voltage of the ML, which was precharged to $V_{\mathrm{DD}}$ before the evaluation, is lowered if at least one cell is a mismatch, while it is maintained if all cells satisfy the match condition.

\section{B. Circuit simulation}

We analyzed the basic operation of the proposed TCAM through SPICE simulation. We assumed that the size of the CoFe-based MTJ was $0.76 \mu \mathrm{m}^{2}$, which resulted in the four TMR values of $4.1,5.4,6.4$, and $10 \mathrm{k} \Omega$. We used three states-4.1, 6.4, and $10 \mathrm{k} \Omega$-among them for three-level data storage. We refer to these three states as $0, \mathrm{X}$, and 1 . The device parameters of the MOSFETs used in the simulation are summarized in Table II.

Figure 8 shows simulated load-line diagrams of (a) the first inverter and (b) the second inverter of the TCAM cell. When the search data are different from the stored data (mismatch), the output voltage of $\mathrm{M} 1\left(V_{\mathrm{D} 1}\right)$ is set to approximately $0.51 \mathrm{~V}$, and a current of approximately $11 \mu \mathrm{A}$ flows through the M2, M3, and M4 transistors, which discharges the ML. On the other hand, the maximum current flowing from the ML under match conditions is less than $100 \mathrm{nA}$. This means that the discharge current ratio between mismatch and match is more than 100. Figure 9 shows a simulated waveform of the ML voltage. The ML was precharged to $V_{\mathrm{DD}}$ before the matching operation. If the input search

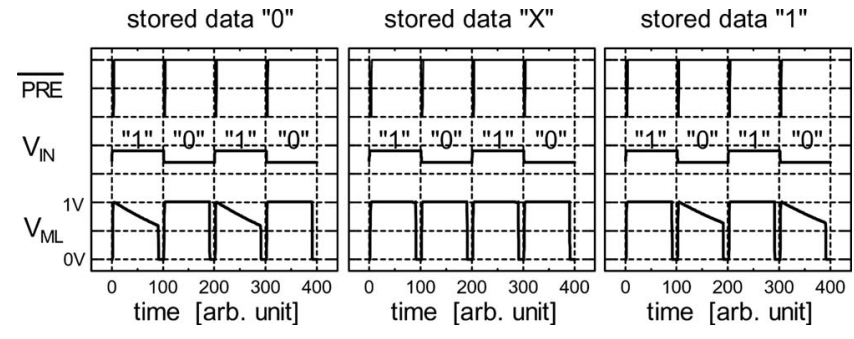

FIG. 9. Simulated waveform of match-line voltage. If the input search data are different from the stored data, the ML voltage is decreased, while it is unchanged if the input data are matched to the stored data. Note that if the stored data is " $\mathrm{X}$," the ML voltage is unchanged regardless of the input data. 
data are different from the stored data, the ML voltage is decreased, while it is unchanged if the input data are matched to the stored data. Note that if the stored data are X, the ML voltage is unchanged regardless of the input data.

\section{SUMMARY}

We have developed three-state MTJs using epitaxial CoFe-based MTJs with a TMR ratio of $145 \%$ at RT. Three TMR values selected from the four TMR values, corresponding to four remanent magnetization states in the singlecrystalline $\mathrm{Co}_{50} \mathrm{Fe}_{50}$ electrode, were separated from each other by more than $56 \%$ at RT. We have shown a novel writing method that enables the magnetization direction of the selected cell to be changed without disturbing those of the half-selected cells by applying the magnetic field along directions $22.5^{\circ}$ from the $\langle 110\rangle$ directions. Furthermore, we proposed a nonvolatile TCAM, which uses a MTJ-based ternary memory for three-level data storage and an inverter with an NDR load for ternary data matching. The proposed TCAM is advantageous in terms of its high density and lowpower operation because it is nonvolatile and device numbers can be reduced to $1 / 3$ that of conventional CMOS-based CAMs.

\section{ACKNOWLEDGMENTS}

This work was partly supported by a Grant-in Aid for Scientific Research (A) (Grant No. 20246054), a Grant-in Aid for Scientific Research on Priority Areas (Grant No. 19048001), a Grant-in-Aid for Scientific Research (C) (Grant No. 19560307), and a Grant-in-Aid from Hokkaido Innovation through Nanotechnology Support (HINTS) from the
Ministry of Education, Culture, Sports, Science and Technology, Japan.

${ }^{1}$ W.-C. Jeong, B. Lee, and S. Joo, IEEE Trans. Magn. 34, 1069 (1998).

${ }^{2}$ W.-C. Jeong, B. Lee, and S. Joo, IEEE Trans. Magn. 35, 2937 (1999).

${ }^{3}$ Y. K. Zheng, Y. H. Wu, Z. B. Guo, G. C. Han, K. B. Li, J. J. Qiu, and P. Luo, IEEE Trans. Magn. 38, 2850 (2002).

${ }^{4}$ C. T. Tanaka, J. Nowak, and J. S. Moodera, J. Appl. Phys. 86, 6239 (1999).

${ }^{5}$ S. P. Parkin, C. Kaiser, A. Panchula, P. M. Rice, B. Hughes, M. Samant, and S.-H. Yang, Nature Mater. 3, 862 (2004).

${ }^{6}$ S. Yuasa, T. Nagahama, A. Fukushima, Y. Suzuki, and K. Ando, Nature Mater. 3, 868 (2004)

${ }^{7}$ D. D. Djayaprawira, K. Tsunekawa, M. Nagai, H. Maehara, S. Yamagata, N. Watanabe, S. Yuasa, Y. Suzuki, and K. Ando, Appl. Phys. Lett. 86, 092502 (2005)

${ }^{8}$ Y. M. Lee, J. Hayakawa, S. Ikeda, F. Matsukura, and H. Ohno, Appl. Phys. Lett. 90, 212507 (2007)

${ }^{9}$ N. Tezuka, N. Ikeda, S. Sugimoto, and K. Inomata, Jpn. J. Appl. Phys., Part 2 46, L454 (2007).

${ }^{10}$ T. Marukame, T. Ishikawa, S. Hakamata, K.-i. Matsuda, T. Uemura, and M. Yamamoto, Appl. Phys. Lett. 90, 012508 (2007).

${ }^{11}$ T. Ishikawa, S. Hakamata, K.-i. Matsuda, T. Uemura, and M. Yamamoto, J. Appl. Phys. 103, 07A919 (2008).

${ }^{12}$ T. Uemura, T. Marukame, K.-i. Matsuda, and M. Yamamoto, IEEE Trans. Magn. 43, 2791 (2007).

${ }^{13}$ T. Hanyu, N. Kanagawa, and M. Kameyama, IEEE J. Solid-State Circuits 31, 1669 (1996).

${ }^{14}$ H. Kimura, H. T. Hanyu, and M. Kameyama, IEEE J. Solid-State Circuits 39, 919 (2004).

${ }^{15}$ T. Hanyu, H. Kimura, and M. Kameyama, Proceedings of the 29th IEEE International Symposium on Multiple-Valued Logic, 1999 (unpublished), p. 30 .

${ }^{16}$ K. Degawa, T. Aoki, T. Higuchi, H. Inokawa, and Y. Takahashi, J. Multiple-Valued Logic and Soft Computing 13, 249 (2007).

${ }^{17}$ K. Degawa, T. Aoki, T. Higuchi, H. Inokawa, K. Nishiguchi, and Y. Takahashi, Proceedings of the 36th IEEE International Symposium on Multiple-Valued Logic (CD-ROM), 2006 (unpublished).

${ }^{18}$ L. O. Chua, J. Yu, and Y. Yu, IEEE Trans. Circuits Syst. 32, 46 (1985). 